\title{
Specificity for interval-valued fuzzy sets
}

\author{
Ramón González-del-Campo ${ }^{1}$, Luis Garmendia ${ }^{2}$, Ronald R. Yager $^{3}$ \\ ${ }^{1}$ DSIC, Universidad Complutense de Madrid, Spain \\ E-mail:rgonzale@estad.ucm.es \\ ${ }^{2}$ DISIA, Universidad Complutense de Madrid, Spain \\ E-mail: lgarmend@fdi.ucm.es \\ ${ }^{3}$ Iona College, USA \\ E-mail: yager@panix.com \\ Received 30 November 2011 \\ Accepted 1 December 2011
}

\begin{abstract}
In this paper some axiomatic definitions about specificity for interval-valued fuzzy sets are proposed. Some examples of measures of specificity for interval-valued fuzzy sets are showed. It is also defined a extension of the notion of alpha cut for interval-valued fuzzy sets and a generalized similarity for intervalvalued fuzzy relations. An axiomatic definition of specificity of interval-valued fuzzy sets under the knowledge of a generalized similarity is given.
\end{abstract}

Keywords: Specificity measure, Interval-valued fuzzy set, Similarity, T-indistinguishability.

\section{Introduction}

Interval-valued fuzzy sets $(\mathscr{I} \mathscr{V} \mathscr{F} \mathscr{S})$ were introduced in the 60 s by Grattan-Guinness ${ }^{7}$, Jahn ${ }^{8}$, Sambuc ${ }^{9}$ and Zadeh ${ }^{16}$. They are extensions of classical fuzzy sets where the membership degree of the elements on the universe of discourse (between 0 and 1$)$ is replaced by an interval in $[0,1] \times[0,1]$. They easily allow to model uncertainty and vagueness generalizing the fuzzy sets. Sometimes it is easier for experts to give a "membership interval" than a membership degree to a characteristic of objects on a universe. $\mathscr{I} \mathscr{V} \mathscr{F} \mathscr{S}$ are a special case of type-2 fuzzy sets that simplifies the calculations while preserving their richness as well.

The concept of specificity provides a measure of the amount of information contained in a fuzzy set. It is strongly related to the inverse of the cardinal- ity of a set. Specificity measures were introduced by Yager ${ }^{10,11}$ showing its usefulness as a measure of tranquility when making a decision. The output information of expert systems and other knowledgebased system should be both specific and correct to be useful.

Measures of specificity have been widely analyzed ${ }^{3,4,5}$, for intuitionistic fuzzy sets ${ }^{14}$, for interval-valued fuzzy sets and for type 2 fuzzy sets 13 .

\section{Preliminaries}

Let $X=\left\{e_{1}, \ldots, e_{n}\right\}$ be a finite set.

Definition 2.1 A fuzzy set $\mu$ on $X$ is normal if there exists an element $x \in X$ such that $\mu(x)=1$.

Definition 2.2 ${ }^{11}$ Let $a_{j}$ be the $j^{\text {th }}$ greatest membership degree of $\mu$. A measure of specificity is a func- 
tion Sp: $\left\{a_{j}\right\} \rightarrow[0,1]$ such that:

- $\operatorname{Sp}(\mu)=1$ if and only if $\mu$ is a singleton.

- $\operatorname{Sp}(\emptyset)=0$

- $S p(\mu)$ depends on $a_{j}$ in that way:

$$
\begin{aligned}
& \text { 1. } \frac{\partial S p(\mu)}{\partial a_{1}}>0 \\
& \text { 2. } \frac{\partial S p(\mu)}{\partial a_{j}} \leqslant 0 \text { for all } j \geqslant 2
\end{aligned}
$$

It is also defined a weaker measure of specificity: Definition 2.3 ${ }^{11}$ Let $[0,1]^{X}$ be the class of fuzzy sets of $X$. A weak measure of specificity is a function Sp: $[0,1]^{X} \rightarrow[0,1]$ such that:

- $\operatorname{Sp}(\mu)=1$ if and only if $\mu$ is a singleton.

- $\operatorname{Sp}(\emptyset)=0$

- If $\mu$ and $\eta$ are normal fuzzy sets in $X$ and $\mu \subset \eta$, then $\operatorname{Sp}(\mu) \geqslant \operatorname{Sp}(\eta)$.

Definition 2.4 Let $S p$ and $S p^{\prime}$ be two measures of specificity. $S p$ is more strict than $S p^{\prime}$, denoted by $S p \leqslant S p^{\prime}$, if for all sets, $\mu$, it verifies: $S p(\mu) \leqslant$ $S p^{\prime}(\mu)$.

Yager introduced ${ }^{11}$ the linear measure of specificity on a finite space $X$ as:

$$
S p_{\vec{w}}(\mu)=a_{1}-\sum_{j=2}^{n} w_{j} a_{j}
$$

where $a_{j}$ is the $j^{t h}$ greatest membership degree of $\mu$ and $\left\{w_{j}\right\}$ is a set of weights verifying:

- $w_{j} \in[0,1]$

- $\sum_{j=2}^{n} w_{j}=1$

- $\left\{w_{j}\right\}$ is not increasing.

Definition 2.5 ${ }^{15}$ A fuzzy relation $R: X^{2} \rightarrow[0,1]$ is a similarity relation if it is reflexive, symmetric and transitive under the t-norm minimum $(\operatorname{Min}(R(a, b), R(b, c)) \leqslant R(a, c)$ for all $a, b, c$ in $X)$.

Yager also a defines a measure of specificity under the knowledge of a similarity to solve the Yager's jacket problem ${ }^{12}$.

Definition 2.6 ${ }^{12}$ Let $\mu$ be a fuzzy set on $X$ and let $S$ be a similarity $S: X \times X \rightarrow[0,1]$. Let $\pi_{\alpha}$ be the set of classes of equivalence of the $\alpha$-cut of $S$. The set of classes of equivalence under the knowledge of $S \mu_{\alpha} / S$ is the subset of equivalence classes of the $\alpha$-cut of $S$ defined in that way: a equivalence class of the $\alpha$-cut of $S$ belongs to $\mu_{\alpha} / S$ if its intersection with the $\alpha$-cut of $\mu_{\alpha}$ is not empty.
Definition 2.7 ${ }^{12}$ Let $[0,1]^{X}$ be the set of fuzzy sets on $X$. Let $\mu$ be a fuzzy set on $X$ and let $S$ be a similarity $S: X \times X \rightarrow[0,1]$. The specificity of $\mu$ under $S$ is defined as follows:

$$
S p(\mu / S)=\int_{0}^{\alpha_{\max }} \frac{1}{\operatorname{card}\left(\mu_{\alpha} / S\right)} d \alpha
$$

Definition $2.8{ }^{2}$ It is denoted by $L$ and $\leqslant_{L}$ the following set and an order relation:

1. $L=\left\{\left[x_{1}, x_{2}\right] \in[0,1]^{2}\right.$ with $\left.x_{1} \leqslant x_{2}\right\}$.

2. $\left[x_{1}, x_{2}\right] \leqslant L\left[y_{1}, y_{2}\right]$ if and only if $x_{1} \leqslant y_{1}$ and $x_{2} \leqslant y_{2}$

Also by definition:

$$
\begin{aligned}
& {\left[x_{1}, x_{2}\right]<_{L}\left[y_{1}, y_{2}\right] \Leftrightarrow x_{1}<y_{1}, x_{2} \leqslant y_{2} \text { or }} \\
& x_{1} \leqslant y_{1}, x_{2}<y_{2} \\
& {\left[x_{1}, x_{2}\right]={ }_{L}\left[y_{1}, y_{2}\right] \Leftrightarrow x_{1}=y_{1}, x_{2}=y_{2} .}
\end{aligned}
$$

$0_{L}={ }_{L}[0,0]$ and $1_{L}={ }_{L}[1,1]$ are the smallest and the greatest elements in $L$ respectively.

$L$ is a complete lattice and the supremum and infimum are defined as follows:

Definition 2.9 ${ }^{1}$ Let $\left\{\left[v_{i}, w_{i}\right]\right\}$ be a set of intervals on L. Then

1. Meet $\left.\left\{\left[v_{i}, w_{i}\right]\right\} \equiv \operatorname{infimun}\left\{v_{i}\right\}, \operatorname{infimun}\left\{w_{i}\right\}\right]$

2. Joint $\left\{\left[v_{i}, w_{i}\right]\right\} \equiv\left[\operatorname{supremun}\left\{v_{i}\right\}, \operatorname{supremun}\left\{w_{i}\right\}\right]$

Definition 2.10 ${ }^{2}$ An interval-valued fuzzy set $A$ on a universe $X$ can be represented by the mapping:

$$
A: X \rightarrow[0,1]^{2}
$$

Definition 2.11 ${ }^{2}$ Let $X$ be a universe and $A$ and $B$ two interval-valued fuzzy sets. The equality between $A$ and $B$ is defined as: $A={ }_{L} B$ if and only if $A(a)={ }_{L} B(a) \forall a \in X$.

Definition $2.12{ }^{2}$ Let $X$ be a universe and $A$ and $B$ two interval-valued fuzzy sets. The inclusion of $A$ in to $B$ is defined as: $A \subseteq_{L} B$ if and only if $A(a) \leqslant_{L} B(a)$ $\forall a \in X$.

Definition 2.13 ${ }^{2}$ An interval-valued negation $\mathscr{N}$ is a decreasing function, $\mathscr{N}: L \rightarrow L$, that satisfies:

$$
\begin{aligned}
& \text { 1. } \mathscr{N}\left(0_{L}\right)={ }_{L} 1_{L} \\
& \text { 2. } \mathscr{N}\left(1_{L}\right)={ }_{L} 0_{L}
\end{aligned}
$$


If $\mathscr{N}\left(\mathscr{N}\left(\left[x_{1}, x_{2}\right]\right)\right)={ }_{L}\left[x_{1}, x_{2}\right]$ then $\mathscr{N}$ is called an involutive negation.

Definition 2.14 A strong interval-valued negation $\mathscr{N}$ is a strictly decreasing and involutive function, $\mathscr{N}: L \rightarrow L$, that satisfies:

$$
\begin{aligned}
& \text { 1. } \mathscr{N}\left(0_{L}\right)={ }_{L} 1_{L} \\
& \text { 2. } \mathscr{N}\left(1_{L}\right)={ }_{L} 0_{L}
\end{aligned}
$$

Example 2.1 Let $\mathscr{N}$ be the involutive mapping defined by:

$$
\begin{aligned}
& \mathscr{N}: L \rightarrow L \\
& \mathscr{N}\left(\left[x_{1}, x_{2}\right]\right)={ }_{L}\left[1-x_{2}, 1-x_{1}\right]
\end{aligned}
$$

Then $\mathscr{N}$ is a negation operator for interval-valued fuzzy sets. It is trivial to prove that: $\mathscr{N}\left(0_{L}\right)={ }_{L} 1_{L}$, $\mathscr{N}\left(1_{L}\right)={ }_{L} 0_{L}$ and $\mathscr{N}\left(\mathscr{N}\left(\left[x_{1}, x_{2}\right]\right)\right)={ }_{L}\left[x_{1}, x_{2}\right]$.

Definition $2.15{ }^{2}$ A generalized t-norm function $\mathscr{T}$ is a monotone increasing, symmetric and associative operator, $\mathscr{T}: L^{2} \rightarrow L$, that satisfies: $\mathscr{T}\left(1_{L},\left[x_{1}, x_{2}\right]\right)={ }_{L}\left[x_{1}, x_{2}\right]$ for all $\left[x_{1}, x_{2}\right]$ in $L$.

Example 2.2 Let Inf $f_{L}$ be defined as follows:

$\operatorname{Inf}_{L}\left(\left[x_{1}, x_{2}\right],\left[y_{1}, y_{2}\right]\right)=\operatorname{Meet}\left\{\left[x_{1}, x_{2}\right],\left[y_{1}, y_{2}\right]\right\}$

It easy to prove that In $f_{L}$ is a generalized $t$-norm.

\section{Specificity for Interval-valued Fuzzy Sets}

Definition 3.1 A operator $G:[0,1]^{n} \rightarrow[0,1]$ is an operator of specificity if it is continuous and it is increasing for the first argument and decreasing for the others and satisfies:

- $G(1,0 \ldots 0)=1$

- $G(0,0 \ldots 0)=0$

Lemma 3.1 Let $\mu$ be a fuzzy set on $X$. Let $\left\{\mu\left(a_{i}\right)\right\}$ for all $i=1 . . n$ the list of membership degrees of $\mu$ decreasing order. Let $G:[0,1]^{n} \rightarrow[0,1]$ be an operator of specificity. Then $G\left(\mu\left(a_{1}\right), \ldots, \mu\left(a_{n}\right)\right)$ is a measure of specificity for $\mathscr{F} \mathscr{S} s$.

Proof. trivial by definition 2.2

Definition 3.2 An operator $f(x, y):[0,1]^{2} \rightarrow[0,1]$ with $x \leqslant y$ is called transformation operator if it is continuous, increasing and verifies:
1. $f(1,1)=1$

2. $f(0,0)=0$

3. $f(0, x)>0$ for all $x \in(0,1]$

4. $f(x, 1)<1$ for all $x \in[0,1)$

Some examples of transformation operators are the following:

Example 3.1

$$
f(x, y)=\frac{x+y}{2}
$$

\section{Example 3.2}

$$
f(x, y)=\alpha * x+\beta * y
$$

with $\alpha+\beta=1, \alpha>0, \beta>0$

Example 3.3

$$
f(x, y)=\frac{x^{2}+y^{2}}{2}
$$

Definition 3.3 Let $\mu$ be an interval-valued fuzzy set on $X$ and let $\left\{\left[x_{1_{q}}, x_{2_{q}}\right]\right\}$ for all $q: 1 . . n$ be its membership intervals. Let $f$ be a transformation operator. Then, the f-list of $\mu$ is the set of all the membership intervals of elements of $X$, ordered decreasingly through the operator $f$, that is, $[x, y] \leqslant f[z, t]$ if and only if $f(x, y) \leqslant f(z, t)$.

Example 3.4 Let $X$ be the universe with cardinality 5 and let $\mu$ be the following interval-valued fuzzy set:

$$
\begin{aligned}
& \mu=\left\{[0.8,0.9] / e_{1},[0.2,0.4] / e_{2},[0.8,1.0] / e_{3},\right. \\
& \left.[0.1,0.2] / e_{4},[0.0,0.1] / e_{5}\right\}
\end{aligned}
$$

Then, if $f(x, y)=(x+y) / 2$ then:

\begin{tabular}{|c|c|}
\hline$[x, y]$ & $f(x, y)$ \\
\hline$[0.8,0.9]$ & 0.85 \\
\hline$[0.2,0.4]$ & 0.30 \\
\hline$[0.8,1.0]$ & 0.90 \\
\hline$[0.1,0.2]$ & 0.15 \\
\hline$[0.0,0.1]$ & 0.05 \\
\hline
\end{tabular}

The f-list of $\mu$ is:

$\{[0.8,1.0],[0.8,0.9],[0.2,0.4],[0.1,0.2],[0.0,0.1]\}$

Definition 3.4 An interval-valued fuzzy set $\mu$ on $X$ is a singleton if there exists an element $a_{i} \in X$ such that $\mu\left(a_{i}\right)=1_{L}$ and $\mu\left(a_{j}\right)=0_{L}$ (for all $j \neq i$ ) for the others. 
Definition 3.5 Let $\left([0,1]^{2}\right)^{X}$ be the set of intervalvalued fuzzy sets on $X$. Let $f$ be a transformation operator. Let $\left\{\left[x_{1_{q}}, x_{2_{q}}\right]\right\}$ for all $q=1 . . n$ be the f-list of $\mu$. A f-measure of specificity for interval-valued fuzzy sets is a function $S p_{f}:\left([0,1]^{2}\right)^{X} \rightarrow[0,1]$ such that:

- $\operatorname{Sp}_{f}(\mu)=1$ if and only if $\mu$ is a singleton.

- $\operatorname{Sp}_{f}(\emptyset)=0$.

- If $\left[x_{1_{1}}, x_{2_{1}}\right]$ increases (according to $\leqslant_{L}$ ) then $S p_{f}(\mu)$ increases.

- If $\left[x_{1_{q}}, x_{2_{q}}\right]$ increases (according to $\leqslant_{L}$ ) then $S p_{f}(\mu)$ decreases for all $q: 2 . . n$.

Definition 3.6 An interval-valued fuzzy set $\mu$ on $X$ is normal if there exists an element $a \in X$ such that $\mu(a)=1_{L}$.

Definition $3.7{ }^{6}$ Let $\left([0,1]^{2}\right)^{X}$ be the set of membership degrees of interval-valued fuzzy sets on $X$. A weak measure of specificity for interval-valued fuzzy sets is a function $\mathrm{Sp}:\left([0,1]^{2}\right)^{X} \rightarrow[0,1]$ such that:

- $\operatorname{Sp}(\mu)=1$ if and only if $\mu$ is a singleton.

- $\operatorname{Sp}(\emptyset)=0$

- If $\mu$ and $\eta$ are normal fuzzy sets in $X$ and $\mu \subseteq_{L} \eta$, then $\operatorname{Sp}(\mu) \geqslant S p(\eta)$.

Lemma 3.2 If $S p_{f}$ is an f-measure of specificity for interval-valued fuzzy sets then $S p_{f}$ is a weak measure of specificity for interval-valued fuzzy sets.

Proof. Let $\left\{\left[x_{1_{q}}, x_{2_{q}}\right]\right\}$ and $\left\{\left[y_{1_{q}}, y_{2_{q}}\right]\right\}$ for all $q=$ $1 . . n$ be the f-list of $\mu$ and $\eta$ respectively. If $\mu$ and $\eta$ are normal and $\mu \subseteq_{L} \eta$ then $\left[x_{1_{q}}, x_{2_{q}}\right] \leqslant_{L}\left[y_{1_{q}}, y_{2_{q}}\right]$ for all $q=2 . . n$. According to the fourth axiom of the definition $3.5 S p_{f}(\mu) \geqslant S p_{f}(\eta)$

Example 3.5 In ${ }^{13}$ Yager shows a particular case of function of transformation, $f$, (called $\left.Q_{F}\right)$. Let $\mu$ be an interval-valued fuzzy set on $X$ with $\mu\left(a_{q}\right)=$ $\left[x_{1_{q}}, x_{2_{q}}\right]$ for all $q: 1 . . n$.

$$
Q_{F}\left(a_{i}\right)=f\left(x_{1_{q}}, x_{2_{q}}\right) \text { such that } x \leqslant f(x, y) \leqslant y \text { for }
$$

Let $a_{i}$ be the element of $X$ which maximizes $Q_{F}$. Then, the following expression is a measure of specificity for interval-valued fuzzy sets:

$$
S p=Q_{F}\left(a_{i}\right)-\frac{1}{n-1} \sum_{\forall k \neq i} Q_{F}\left(a_{k}\right) .
$$

Lemma 3.3 Let $\mu$ be an interval-valued fuzzy set on $X$ and let $S p_{f}$ be any f-measure of specificity over $\mu$. Let $\left\{\left[x_{1_{q}}, x_{2_{q}}\right]\right\}$ for all $q: 1 . . n$ the f-list of $\mu$. Then, there exists an operator of specificity $G:[0,1]^{n} \rightarrow[0,1]$ such that:

$$
S p_{f}(\mu)=G\left(f\left(x_{1_{1}}, x_{2_{1}}\right), \ldots, f\left(x_{1_{n}}, x_{2_{n}}\right)\right)
$$

Corollary 3.1 Let $G$ be a measure of specificity for $\mathscr{F} \mathscr{S} s$. Let $f$ a transformation operator. Then $G\left(f\left(x_{1_{1}}, x_{2_{1}}\right), \ldots, f\left(x_{1_{n}}, x_{2_{n}}\right)\right)$ is a $f$-measure for $\mathscr{I} \mathscr{V} \mathscr{F} \mathscr{S} S$

Definition 3.8 Let $S p_{f}$ and $S p_{g}^{\prime}$ be two measures of specificity. $S p_{f}$ is more strict than $S p_{g}^{\prime}$, denoted by $S p_{f} \leqslant S p_{g}^{\prime}$, if for all set, $\mu$, it verifies: $S p_{f}(\mu) \leqslant$ $S p_{g}^{\prime}(\mu)$.

Theorem 3.1 $S p_{f}$ is more strict than $S p_{g}^{\prime}$ if and only if $f(x, y) \leqslant g(x, y)$ for all $x, y$.

Proof. Trivial

Theorem 3.2 Let $f$ be a transformation operator and $\left\{\alpha_{i}\right\}$ a set of weights that satisfies:

- $\alpha_{j} \in(0,1]$

- $\sum_{j=2}^{n} \alpha_{j}=1$

- $\left\{\alpha_{j}\right\}$ is not increasing.

Let $T, T^{\prime}$, $S$ and $N$ be, two t-norms, a t-conorm and a negation (in $[0,1], \leqslant)$ respectively. Let $\left\{f\left(x_{1_{k}}, x_{2_{k}}\right)\right\}$ be the $f$-list of an interval-valued fuzzy set $\mu$. Then

$$
\begin{gathered}
S p_{f}(\mu)=T\left(f\left(x_{1_{1}}, x_{2_{1}}\right){ }_{1}, N\left(S\left(T^{\prime}\left(\alpha_{2}, f\left(x_{1_{2}}, x_{2_{2}}\right)\right)\right), \ldots\right.\right. \\
\left.\left.\left.\ldots, T\left(\alpha_{n}, f\left(x_{1_{n}}, x_{2_{n}}\right)\right)\right)\right)\right)
\end{gathered}
$$

is a f-measure of specificity for interval-valued fuzzy set.

This expression is a generalization of the t-norm based measure of specificity given in ${ }^{3}$ but extended for $\mathscr{I} \mathscr{V} \mathscr{F} \mathscr{S}$.

\section{Proof.}

1. $S p_{f}(\mu)=1$ if and only if $\mu$ is a singleton:

- If $\mu$ is a singleton then $\left[x_{1_{1}}, x_{2_{1}}\right]=[1,1]$ and $\left[x_{1}, x_{2_{k}}\right]=[0,0]$ for all $k>1$. Then $f\left(x_{1_{1}}, x_{2_{1}}\right)=1$ and $f\left(x_{1_{k}}, x_{2_{k}}\right)=0$ for all $k>1$. 
- If $S p_{f}(\mu)=1$, it is necessary that $f\left(x_{1_{1}}, x_{2_{1}}\right)=1$ and

$S\left(T\left(\alpha_{2}, f\left(x_{1_{2}}, x_{2_{2}}\right)\right)\right), \ldots, T\left(\alpha_{n}, f\left(x_{1_{n}}, x_{2_{n}}\right)\right)=0$

Then $\left.T\left(\alpha_{k}, f\left(x_{1_{k}}, x_{2_{k}}\right)\right)\right)=0$ for all $k$ and $f\left(x_{1_{k}}, x_{2_{k}}\right)=0$ for all $k$.

2. $S p_{f}(\emptyset)=0$ : trivial.

3. Trivial due to the fact $T, T^{\prime}$ and $S$ are monotonic

Let $\left\{\alpha_{i}\right\}$ be a set of weights which satisfies the conditions of theorem 3.2.

Example 3.6 With $T(a, b)=\operatorname{Max}\{0, a+b-1\}$, $N(a)=1-a$,

$S(a, b)=\operatorname{Min}\{1, a+b\}$,

$T^{\prime}(a, b)=a * b$ and $f(x, y)=\frac{x+y}{2}$, it is obtained:

$$
S p_{f}(\mu)=\frac{1}{2}\left(x_{1_{1}}+x_{2_{1}}\right)-\sum_{j=2}^{n} \alpha_{j}\left(x_{1_{j}}+x_{2_{j}}\right)
$$

Example 3.7 With $T(a, b)=\operatorname{Max}\{0, a+b-1\}$, $N(a)=1-a$,

$S(a, b)=\operatorname{Min}\{1, a+b\}$,

$T^{\prime}(a, b)=a * b$ and $f(x, y)=\alpha * x+\beta * y$ with $\alpha+\beta=1, \alpha>0, \beta>0$, it is obtained:

$S p_{f}(\mu)=\alpha * x_{1_{1}}+\beta * x_{2_{1}}-\sum_{j=2}^{n} \alpha_{j}\left(\alpha * x_{1_{j}}+\beta * x_{2_{j}}\right)$

Example 3.8 With $T(a, b)=\operatorname{Max}\{0, a+b-1\}$, $N(a)=1-a$,

$S(a, b)=\operatorname{Min}\{1, a+b\}$,

$T^{\prime}(a, b)=a * b$ and $f(x, y)=\frac{x^{2}+y^{2}}{2}$, it is obtained:

$$
S p_{f}(\mu)=\frac{1}{2}\left(x_{1_{1}}^{2}+x_{2_{1}}^{2}\right)-\frac{1}{2} \sum_{j=2}^{n} \alpha_{j} *\left(x_{1_{j}}^{2}+x_{2_{j}}^{2}\right)
$$

Examples 3.6 and 3.7 are extensions of R. Yager's linear measure of specificity ${ }^{11}$ for $\mathscr{I} \mathscr{V} \mathscr{F} \mathscr{S}$.

\section{Alpha cuts for interval-valued fuzzy sets}

Definition 4.1 Let $\mu$ be an interval-valued fuzzy set on $X$. The $\alpha_{1}, \alpha_{2}$ cuts of $\mu$ are subsets of $X$ defined as follows:

$$
\mu_{\alpha_{1}, \alpha_{2}}=\left\{a_{i} \mid \mu\left(a_{i}\right) \geqslant_{L}\left[\alpha_{1}, \alpha_{2}\right]\right\}
$$

Definition 4.2 Let $R$ be an interval-valued relation $R: X^{2} \rightarrow L$. The $\alpha_{1}, \alpha_{2}$ cut of $R, R_{\alpha_{1}, \alpha_{2}}$, is a crisp relation defined for all $\alpha_{1}, \alpha_{2}$ in $[0,1]$ as follows:

$$
R_{\alpha_{1}, \alpha_{2}}\left(a_{i}, a_{j}\right)= \begin{cases}1 & R\left(a_{i}, a_{j}\right) \geqslant_{L}\left[\alpha_{1}, \alpha_{2}\right] ; \\ 0, & \text { otherwise. }\end{cases}
$$

Lemma 4.1 Let $R=\left[R_{\text {down }}, R_{\text {up }}\right]$ be an intervalvalued fuzzy relation on $X$ where $R_{\text {down }}$ and $R_{u p}$ are fuzzy relations on $X$, it is, $R\left(a_{i}, a_{j}\right)=$ $\left[R_{\text {down }}\left(a_{i}, a_{j}\right), R_{\text {up }}\left(a_{i}, a_{j}\right)\right]$ for all $a_{i}, a_{j}$ in $X$. Then, $R_{\alpha_{1}, \alpha_{2}}\left(a_{i}, a_{j}\right)=1$ if and only if $R_{\text {down } \alpha_{1}}\left(a_{i}, a_{j}\right)=1$ and $R_{\text {up } \alpha_{2}}\left(a_{i}, a_{j}\right)=1$

Proof. Trivial due to definition 4.2

Lemma 4.2 Let $R, S$ be two fuzzy relations. If $R_{\alpha}\left(a_{i}, a_{j}\right)=S_{\alpha}\left(a_{i}, a_{j}\right)$ for all $a_{i}, a_{j}$ on $X$ and for all $\alpha$ in $[0,1]$ then $R\left(a_{i}, a_{j}\right)=S\left(a_{i}, a_{j}\right)$.

Proof. Let's suppose that there exist $r, s$ such that: $R\left(a_{r}, a_{s}\right) \neq S\left(a_{r}, a_{s}\right)$. If $R\left(a_{r}, a_{s}\right)>S\left(a_{r}, a_{s}\right)$ then $R_{p}\left(a_{r}, a_{s}\right)=1$ and $S_{p}\left(a_{r}, a_{s}\right)=0$ where $p=R\left(a_{r}, a_{s}\right)$ which is a contradiction. If $R\left(a_{r}, a_{s}\right)<S\left(a_{r}, a_{s}\right)$ a similar contradiction can be found.

Proposition 4.1 The set of all $\alpha_{1}, \alpha_{2}$ cuts of an interval-valued fuzzy relation $R$ determine $R$.

Proof. By lemma 4.1 the $\alpha_{1}, \alpha_{2}$ cuts of an intervalvalued fuzzy relation $R$ are determined by the $\alpha$ cuts of $R_{\text {down }}$ and the $\alpha$ cuts of $R_{u p}$, which by lemma 4.2 are determined by the fuzzy relations $R_{\text {down }}$ and $R_{u p}$, that define $R=\left[R_{\text {down }}, R_{u p}\right]$, so the $\alpha_{1}, \alpha_{2}$ cuts of $R$ determine $R$.

Corollary 4.1 Let $R, S$ be two interval-valued fuzzy relations. If $R_{\alpha_{1}, \alpha_{2}}\left(a_{i}, a_{j}\right)=S_{\alpha_{1}, \alpha_{2}}\left(a_{i}, a_{j}\right)$ for all $a_{i}, a_{j}$ on $X$ and for all $\alpha_{1}, \alpha_{2}$ in $[0,1]$ then $R\left(a_{i}, a_{j}\right)=$ $S\left(a_{i}, a_{j}\right)$.

Proof. Trivial due to proposition 4.1

Definition 4.3 Let $\mathscr{T}$ be a generalized $t$-norm ${ }^{2}$. An interval-valued relation $R: X^{2} \rightarrow L$ is a generalized $\mathscr{T}$-indistinguishability if it is reflexive, symmetric and $\mathscr{T}$-transitive, it is:

1. $R(a, a)={ }_{L} 1_{L}$ for all $a$ in $X$.

2. $R(a, b)={ }_{L} R(b, a)$ for all $a, b$ in $X$. 
3. $\mathscr{T}(R(a, b), R(b, c)) \leqslant{ }_{L} R(a, c)$ for all $a, b, c$ in $X$.

Lemma 4.3 Let $R: X^{2} \rightarrow L$ be a generalized $\operatorname{In} f_{L^{-}}$ indistinguishability. Then, for each $\alpha_{1}, \alpha_{2}, R_{\alpha_{1}, \alpha_{2}}$ is an equivalence relation.

Proof.

1. $R_{\alpha_{1}, \alpha_{2}}\left(a_{i}, a_{i}\right)=1$ trivially.

2. $R_{\alpha_{1}, \alpha_{2}}\left(a_{i}, a_{j}\right)=R_{\alpha_{1}, \alpha_{2}}\left(a_{j}, a_{i}\right)$ trivially.

3. Due to the fact that $R$ is a $I n f_{L^{-}}$ indistinguishability:

$$
\begin{gathered}
\operatorname{Inf}_{L}\left(R\left(a_{i}, a_{k}\right), R\left(a_{k}, a_{j}\right)\right) \leqslant_{L} R\left(a_{i}, a_{j}\right) \text { for all } \\
a_{i}, a_{j}, a_{k} \text { in } X
\end{gathered}
$$

If $R_{\alpha_{1}, \alpha_{2}}\left(a_{i}, a_{k}\right)=1$ and $R_{\alpha_{1}, \alpha_{2}}\left(a_{k}, a_{j}\right)=1$ then $R\left(a_{i}, a_{k}\right) \geqslant_{L}\left[\alpha_{1}, \alpha_{2}\right]$ and $R\left(a_{k}, a_{j}\right) \geqslant_{L}$ $\left[\alpha_{1}, \alpha_{2}\right]$ and

$$
\begin{gathered}
{\left[\alpha_{1}, \alpha_{2}\right] \leqslant_{L} \operatorname{Inf}_{L}\left(R\left(a_{i}, a_{k}\right), R\left(a_{k}, a_{j}\right)\right) \leqslant L} \\
R\left(a_{i}, a_{j}\right)
\end{gathered}
$$

therefore: $\left[\alpha_{1}, \alpha_{2}\right] \leqslant{ }_{L} R\left(a_{i}, a_{j}\right)$ and so $R_{\alpha_{1}, \alpha_{2}}$ is transitive

Lemma 4.4 Let $R: X^{2} \rightarrow L$ be an interval-valued relation. If for each $\alpha_{1}, \alpha_{2}, R_{\alpha_{1}, \alpha_{2}}$ is an equivalence relation, then $R$ is a In $f_{L}$-indistinguishability.

\section{Proof.}

1. $R\left(a_{i}, a_{j}\right)=1$ by contradiction.

2. $R\left(a_{i}, a_{j}\right)=R\left(a_{j}, a_{i}\right)$ by contradiction.

3. It is supposed that $R$ is not a $\operatorname{In} f_{L^{-}}$ indistinguishability:

$$
\begin{gathered}
\operatorname{Inf}_{L}\left(R\left(a_{i}, a_{k}\right), R\left(a_{k}, a_{j}\right)\right)>_{L} R\left(a_{i}, a_{j}\right) \text { for } \\
\text { some } a_{i}, a_{j}, a_{k} \text { in } X
\end{gathered}
$$

Then, it is found a $R_{\alpha_{1}, \alpha_{2}}$ that is not a equivalence relation: Let $\varepsilon$ and $\delta$ be two real number arbitrarily small such that $\alpha_{1}=R\left(a_{i}, a_{j}\right)-\varepsilon$ and $\alpha_{2}=\overline{R\left(a_{i}, a_{j}\right)}-\delta$. Then $R_{\alpha_{1}, \alpha_{2}\left(a_{i}, a_{k}\right)}=$ 1 and $R_{\alpha_{1}, \alpha_{2}}\left(a_{k}, a_{j}\right)=1$ but $R_{\alpha_{1}, \alpha_{2}}\left(a_{i}, a_{j}\right)=0$, i.e $R_{\alpha_{1}, \alpha_{2}}$ is not transitive
Theorem 4.1 Let $R: X^{2} \rightarrow L$ be an interval-valued relation. If for each $\alpha_{1}, \alpha_{2}, R_{\alpha_{1}, \alpha_{2}}$ is an equivalence relation if and only if $R$ is a $\operatorname{Inf}_{L}$-indistinguishability.

Proof. Trivial due to the lemmas 4.3 and 4.4

Corollary 4.2 Let $R: X^{2} \rightarrow L$ be an interval-valued relation. Then, $R$ is a In $f_{L^{-}}$-indistinguishability if and only if $R_{\alpha_{1}, \alpha_{2}}$ and $\overline{R_{\alpha_{1}, \alpha_{2}}}$ are equivalence relations for all $\frac{\alpha_{1}, \alpha_{2}}{\alpha_{1}}$.

Theorem 4.2 Let $R: X^{2} \rightarrow L$ be a generalized $\mathscr{T}$ indistinguishability (with $\mathscr{T} \neq \operatorname{In} f_{L}$ ). Then, there exists some $\alpha_{1}, \alpha_{2}$, such that $R_{\alpha_{1}, \alpha_{2}}$ is not an equivalence relation.

Proof. Let $R: X^{2} \rightarrow L$ be a generalized $\mathscr{T}$-indistinguishability (with $\mathscr{T} \neq \operatorname{Inf} f_{L}$ ). Let $a_{i}, a_{j}, a_{k}$ be elements of the universe $X$ such that: $\mathscr{T}\left(R\left(a_{i}, a_{k}\right), R\left(a_{k}, a_{j}\right)\right)={ }_{L} R\left(a_{i}, a_{j}\right)$. Let $\left[\alpha_{1}, \alpha_{2}\right]$ be such that: $\left[\alpha_{1}, \alpha_{2}\right]=\operatorname{Inf} f_{L} R\left(a_{i}, a_{k}\right), R\left(a_{k}, a_{j}\right)$. Then, due the fact that $I n f_{L}$ is the greatest of the generalized t-norms :

$$
\begin{gathered}
R\left(a_{i}, a_{j}\right)=\mathscr{T}\left(R\left(a_{i}, a_{k}\right), R\left(a_{k}, a_{j}\right)\right) \leqslant L \\
\operatorname{Inf}_{L} R\left(a_{i}, a_{k}\right), R\left(a_{k}, a_{j}\right)
\end{gathered}
$$

Then $R_{\alpha_{1}, \alpha_{2}}\left(a_{i}, a_{k}\right)=1, \quad R_{\alpha_{1}, \alpha_{2}}\left(a_{k}, a_{j}\right)=1$ but $R_{\alpha_{1}, \alpha_{2}}\left(a_{i}, a_{j}\right)=0$

\section{Specificity for Interval-valued Fuzzy Sets under generalized similarities}

Proposition 5.1 Let $\mu$ be an interval-valued fuzzy set on $X$. Let $\left[\widehat{\alpha_{1}}, \widehat{\alpha_{2}}\right]=$ Joint $\left\{\mu\left(a_{i}\right)\right\}$ for all i:1..n. Then:

$$
\begin{gathered}
S p(\mu)=2 * \int_{0}^{\widehat{\alpha_{2}}} \int_{0}^{\alpha_{2}} \frac{1}{\operatorname{card}\left(\mu_{\alpha_{1}, \alpha_{2}}\right)} d \alpha_{1} d \alpha_{2}+ \\
\int_{\widehat{\alpha_{1}}}^{\widehat{\alpha_{2}}} \int_{0}^{\widehat{\alpha_{1}}} \frac{1}{\operatorname{card}\left(\mu_{\alpha_{1}, \alpha_{2}}\right)} d \alpha_{1} d \alpha_{2}
\end{gathered}
$$

It is a measure of specificity for $\mathscr{I} \mathscr{V} \mathscr{F} \mathscr{S} s$.

Note that the integration area guarantees that $\operatorname{card}\left(\mu_{\alpha_{1}, \alpha_{2}}\right)$ is not zero.

Proof.

- Axiom 1:

1. If $\mu$ is a singleton then $\operatorname{Sp}(\mu / S)=1$ : 
- Let $a_{k}$ be the only element on $X$ such that $\mu\left(a_{k}\right)=1_{L}$.

- Then $\mu_{\alpha_{1}, \alpha_{2}}=a_{k}$ for all $\alpha_{1}, \alpha_{2}$ and $\operatorname{card}\left(\mu_{\alpha_{1}, \alpha_{2}}\right)=1$ for all $\alpha_{1}, \alpha_{2}$ and $\left[\widehat{\alpha_{1}}, \widehat{\alpha_{2}}\right]=[1,1]$.

- Then

$$
2 * \int_{0}^{1} \int_{0}^{\alpha_{2}} 1 d \alpha_{1} d \alpha_{2}=1
$$

2. So that $\operatorname{Sp}(\mu)=1$ it is necessary that $\left[\widehat{\alpha_{1}}, \widehat{\alpha_{2}}\right]=[1,1]$ and $\operatorname{card}\left(\mu_{\alpha_{1}, \alpha_{2}}\right)=1$. Otherwise $\operatorname{Sp}(\mu)<1$. Hence $\mu$ is a singleton.

- Axiom 2:

Trivial.

- Axiom 3: Let $\left\{\left[x_{1_{q}}, x_{2_{q}}\right]\right\}$ for all $q=1 . . n$ be the f-list of $\mu$.

1. If $\left[x_{1_{1}}, x_{2_{1}}\right]$ increases then $\left[\widehat{\alpha_{1}}, \widehat{\alpha_{2}}\right]$ increases and $\operatorname{card}\left(\mu_{\alpha_{1}, \alpha_{2}}\right)$ does not change.

2. If $\left[x_{1_{q}}, x_{2_{q}}\right]$ for all $q: 2 . . n$ increases then $1 / \operatorname{card}\left(\mu_{\alpha_{1}, \alpha_{2}}\right)$ decreases

In ${ }^{4}$ a set of axioms that generalize the specificity of a fuzzy set under T-indistinguishabilities is given. Definition 5.1 ${ }^{4}$ Let $S p$ a measure of specificity for $\mathscr{I} \mathscr{V} \mathscr{F} \mathscr{S} s . S p(\mu / S)$ is a measure of specificity under a generalized similarity $S$ if it verifies:

1. $\operatorname{Sp}(\mu / S)=1$ if and only if $\mu$ is a singleton.

2. $S p(\emptyset / S)=0$.

3. $\operatorname{Sp}(\mu / I d)=S p(\mu)$.

4. $S p(\mu / S) \geqslant S p(\mu)$.

Definition 5.2 An interval-valued relation $R$ : $X^{2} \rightarrow L$ is a generalized similarity if it is reflexive, symmetric and Inf $f_{L}$-transitive where $\operatorname{Inf}_{L}\left(\left[x_{1}, x_{2}\right],\left[y_{1}, y_{2}\right]\right)=\left[\min \left(x_{1}, y_{1}\right), \min \left(x_{2}, y_{2}\right)\right]$, it is, $R$ is an In $_{L}$-indistinguishability.

Definition 5.3 Let $\mu$ be a fuzzy set on $X$ and let $S$ be a similarity $S: X \times X \rightarrow[0,1]$. Let $\pi_{\alpha_{1}, \alpha_{2}}$ be the set of classes of equivalence of the $\alpha_{1}, \alpha_{2}$ cut of $S$. The set of classes of equivalence under the knowledge of $S \mu_{\alpha_{1}, \alpha_{2}} / S$ is the subset of equivalence classes of the $\alpha_{1}, \alpha_{2}$ cut of $S$ defined in that way: a equivalence class of the $\alpha_{1}, \alpha_{2}$ cut of $S$ belongs to $\mu_{\alpha_{1}, \alpha_{2}} / S$ if its intersection with $\mu_{\alpha_{1}, \alpha_{2}}$ is not empty.
Example 5.1 Let $E=\left\{e_{1}, e_{2}, e_{3}, e_{4}\right\}$. Let $\mu=$ $\left\{[0.6,0.8] / e_{1}+[0.7,0.8] / e_{2}+[0.8,0.8] / e_{3}+\right.$ $\left.[0.9,1.0] / e_{4}\right\}$ and

$$
\begin{gathered}
S=\left(\begin{array}{cccc}
1 & 0.1 & 0.1 & 0.1 \\
0.1 & 1 & 0.8 & 0.6 \\
0.1 & 0.8 & 1 & 0.6 \\
0.1 & 0.6 & 0.6 & 1
\end{array}\right) \\
R_{0.7,0.8}=\left(\begin{array}{llll}
1 & 0 & 0 & 0 \\
0 & 1 & 1 & 0 \\
0 & 1 & 1 & 0 \\
0 & 0 & 0 & 1
\end{array}\right)
\end{gathered}
$$

Then, $\pi_{0.7,0.8}=\left\{\left\{e_{1}\right\},\left\{e_{2}, e_{3}\right\},\left\{e_{4}\right\}\right\} \quad \mu_{0.7,0.8}=$ $\left\{e_{2}, e_{3}, e_{4}\right\}$ and $\pi_{0.7,0.8} / S=\left\{\left\{e_{2}, e_{3}\right\},\left\{e_{4}\right\}\right\}$

Proposition 5.2 Let $\mu$ be an interval-valued fuzzy set on $X$ and let $S$ be a similarity $S: X \times X \rightarrow[0,1]$. Then:

$$
\begin{aligned}
& \operatorname{Sp}(\mu / S)=2 * \int_{0}^{\widehat{\alpha_{2}}} \int_{0}^{\alpha_{2}} \frac{1}{\operatorname{card}\left(\mu_{\alpha_{1}, \alpha_{2} / S}\right)} d \alpha_{1} d \alpha_{2}+ \\
& \int \frac{\widehat{\alpha_{2}}}{\widehat{\alpha_{1}}} \int_{0}^{\widehat{\alpha_{1}}} \frac{1}{\operatorname{card}\left(\mu_{\alpha_{1}, \alpha_{2} / S}\right)} d \alpha_{1} d \alpha_{2}
\end{aligned}
$$

It is a measure of specificity for $\mathscr{I} \mathscr{V} \mathscr{F} \mathscr{S} s$.

Note that the integration area guarantees that $\operatorname{card}\left(\mu_{\alpha_{1}, \alpha_{2} / S}\right)$ is not zero.

Proof. Let $\left\{\pi_{\alpha_{1}, \alpha_{2}}^{i}\right\}$ for all $i$ be the set of equivalence classes of $\pi_{\alpha_{1}, \alpha_{2}}$.

- Axiom 1:

1. If $\mu$ is a singleton then $\operatorname{Sp}(\mu / S)=1$ :

- Let $a_{k}$ be the only element on $X$ such that $\mu\left(a_{k}\right)=1_{L}$.

- Then $\mu_{\alpha_{1}, \alpha_{2}}=a_{k}$ for all $\alpha_{1}, \alpha_{2}$.

- There exists only a $\pi_{\alpha_{1}, \alpha_{2}}^{i}$ such that $a_{k}$ belongs to it.

- And $\operatorname{card}\left(\mu_{\alpha_{1}, \alpha_{2}} / S\right)=1$ for all $\alpha_{1}, \alpha_{2}$.

- Then

$$
2 * \int_{0}^{1} \int_{0}^{\alpha_{2}} 1 d \alpha_{1} d \alpha_{2}=1
$$

2. If $\operatorname{Sp}(\mu / S)=1$ then $\mu$ is a singleton:

If $S p(\mu / S)=1$ then $\operatorname{card}\left(\mu_{\alpha_{1}, \alpha_{2}} / S\right)=1$ for all $\alpha_{1}, \alpha_{2}$ and $\mu$ is a singleton.

- Axiom 2:

Trivial. 
- Axiom 3:

Remember that $X=\left\{a_{1}, \ldots, a_{n}\right\}$, then if $R$ is the relation identity then $\left\{\pi_{\alpha_{1}, \alpha_{2}}^{i}\right\}=a_{i}$ for all $i: 1 . . n$ and $\operatorname{card}\left(\mu_{\alpha_{1}, \alpha_{2}} / S\right)=\operatorname{card}\left(\mu_{\alpha_{1}, \alpha_{2}}\right)$.

- Axiom 4:

For a relation $S$ there will exist $\alpha_{1}, \alpha_{2}$ such that $\operatorname{card}\left(\pi_{\alpha_{1}, \alpha_{2}}^{i}\right)>1$ and $\operatorname{card}\left(\mu_{\alpha_{1}, \alpha_{2}} / S\right)<$ $\operatorname{card}\left(\mu_{\alpha_{1}, \alpha_{2}}\right)$

\section{Conclusion}

Several expression for t-norm based measure of specificity for $\mathscr{I} \mathscr{V} \mathscr{F} \mathscr{S} s$ have been proposed and studied.

An generalized expression for measures of specificity have been proposed for $\mathscr{I} \mathscr{V} \mathscr{F} \mathscr{S} s$ and the measures of specificity under the knowledge of generalized similarities have also been defined following the Yager's jacket ideas.

\section{Acknowledgment}

This research is partially supported by the Spanish Ministry of Science and Technology, grant number TIN2009-07901, the Research Group CAM GR58/08 at Complutense University of Madrid.

\section{References}

1. C. Cornelis, G. Deschrijver, and E. Kerre. Advances and challenges in interval-valued fuzzy logic. Fuzzy Sets and Systems, 157(5):622-627, 2006.

2. C. Cornelis, G.Deschrijver, and E. Kerre. Implication in intuitionistic fuzzy and interval-valued fuzzy set theory: construction, classification, application. Int. J. Approx. Reasoning, 35(1):55-95, 2004.

3. L. Garmendia, R.R. Yager, E. Trillas, and A. Salvador. On t-norms based specificity measures. Fuzzy Sets and Systems, 133(2):237-248, 2003.
4. L. Garmendia, R.R. Yager, E. Trillas, and A. Salvador. General measures of specificity of fuzzy sets under t-indistinguishabilities. IEEE Transactions on Fuzzy Systems, 14(4):568-572, 2006.

5. L. Garmendia, R.R. Yager, E. Trillas, and A. Salvador. A t-norm based specificity for fuzzy sets on compact domains. International Journal of General Systems, 35(6):687-698, 2006.

6. R. González-del Campo and L. Garmendia. Specificity, uncertainty and entropy measures of intervalvalued fuzzy sets. Proceedings EUROFUSE Workshop Preference Modelling and Decision Analysis, pages 273-278, 2009.

7. I. Grattan-Guiness. Fuzzy membership mapped onto interval and many-valued quantities. Math. Logik. Grundladen Math, 22:149-160, 1975.

8. K.U. Jahn. Intervall-wertige mengen. Math. Nach., 68:115-132, 1975.

9. E. Sanchez and R. Sambuc. Fuzzy relationships. phi -fuzzy functions. application to diagnostic aid in thyroid pathology. Proceedings of an International Symposium on Medical Data Processing, pages 513-524, 1976.

10. R.R. Yager. Measuring tranquility and anxiety in decision-making - an application of fuzzy-sets. International Journal of General Systems, 8:139-146, 1982.

11. R.R. Yager. Ordinal measures of specificity. International Journal of General Systems, 17:57-72, 1990.

12. R.R. Yager. Similarity based measures of specificity. International Journal of General Systems, 19:91-106, 1991.

13. R.R. Yager. Containment and specificity for type-2 fuzzy sets. International Journal Of Fuzzy Systems, 9:55-66, 2007.

14. R.R. Yager. On the measure of specificity of intuitionistic fuzzy sets. NAFIPS 2008 - Annual Meeting of the North American Fuzzy Information Processing Society, pages 677-682, 2008

15. L. A. Zadeh. Similarity relations and fuzzy orderings. Inform. Sci. 3, pages 177-200, 1971.

16. L.A. Zadeh. The concept of a linguistic variable and its application to approximate reasoning i. Information Sciences, 8:199-249, 1975. 